\title{
Performance Evaluation of Fully Adaptive Routing for the Torus Interconnect Networks
}

\author{
F. Safaei ${ }^{1,3}$, A. Khonsari ${ }^{2,1}$, M. Fathy ${ }^{3}$, and M. Ould-Khaoua ${ }^{4}$ \\ ${ }^{1}$ IPM School of Computer Science, Tehran, Iran \\ ${ }^{2}$ Dept. of ECE, Univ. of Tehran, Tehran, Iran \\ ${ }^{3}$ Dept. of Computer Eng., Iran Univ. of Science and Technology, Tehran, Iran \\ ${ }^{4}$ Dept. of Computing Science, Univ. of Glasgow, UK \\ \{safaei,ak\}@ipm.ir,\{f_safaei,mahfathy\}@iust.ac.ir, \\ mohamed@dcs.gla.ac.uk
}

\begin{abstract}
Adaptive routing algorithms have been frequently suggested as a means of improving communication performance in parallel computer networks. These algorithms, unlike deterministic routing, can utilize network state information to exploit the presence of multiple paths. Before such schemes can be successfully incorporated in networks, it is necessary to have a clear understanding of the factors which affect their performance potential. This paper proposes a new analytical model to obtain message latency in wormhole-switched 2-D torus interconnect networks. The analysis focuses on a fully adaptive routing which has been shown to be one of the most effective in torus networks. The validity of the model is demonstrated by comparing analytical results with those obtained through simulation experiments.
\end{abstract}

Keywords: adaptive routing algorithm, parallel computer network, torus Interconnect Networks.

\section{Introduction}

Large-scale massively parallel computers, Multiprocessors System-on-Chip (MPSoCs), and peer-to-peer communication networks provide high performance computing that allows users to deal with large and heavy computational tasks. Most of the contemporary massively parallel computers use wormhole switching (also widely known as wormhole routing [1, 2]) mechanism to support their interprocess communication. In the wormhole switching, a message is divided into a sequence of fixedsize units of data, called flits. If a communication channel transmits the first flit of a message, it must transmit all the remaining flits of the same message before transmitting flits of another message. The main drawback of wormhole switching is that blocked messages remain in the network, therefore they waste channel bandwidth and block other messages.

The routing algorithm specifies how a message selects a path to cross from the source to destination, and has great impact on network performance. Many practical systems have used deterministic routing [1, 2] with virtual channels to ensure deadlock avoidance. This is achieved by forcing messages to visit the virtual channels in a 
strict order. This form of routing has the advantage of being simple; however, if any channel along the message path is heavily loaded, the message experiences large delays and if any node or channel along the path is faulty, the message cannot be delivered at all. Alternatively, adaptive routing improves both the performance and, more importantly, fault-tolerance of an interconnect network.

Message passing in massively parallel computers is implemented based on a routing algorithm that determines the path a message follows to reach its destination. Routing algorithms for these systems are generally classified as being either deterministic or adaptive [1]. In deterministic routing, all messages between a given source/destination pair will follow the same path. One of the main benefits is that inorder arrival of messages is preserved. However, deterministic routing usually makes an inefficient use of the network resources [1,3]. An alternative, but complementary approach, to improve network performance consists of using adaptive routing. This routing strategy provides alternative paths to route messages, thus avoiding congested regions in the network and increasing throughput.

This paper proposes an analytical approach to investigate the performance behavior of a fully adaptive routing algorithm in wormhole-switched 2-D torus fortified with a routing scheme suggested in [4], as an instance of routing methodology widely used in the literature to achieve high adaptivity.

The rest of this paper is structured as follows. Section 2 briefly describes node structure, routing scheme, on which the present study is based. Section 3 gives an overview of the assumptions and describes the proposed analytical model. Section 4 compares the delays predicted by analytical model with those obtained through simulation experiments. Finally, Section 5 summarizes our findings and concludes the paper.

\section{Node Structure in the Torus Networks}

A $k$-ary 2-cube (2-D torus) is a direct network with $N=k^{2}$ nodes; $k$ is called the radix. Links (channels) in the torus can be either uni- or bi-directional. Each node can be identified by a 2-digit radix $k$ address $\left(a_{1}, a_{2}\right)$. Nodes, with address $\left(a_{1}, a_{2}\right),\left(b_{1}, b_{2}\right)$ are connected if and only if $a_{1}=\left(a_{2}+1\right) \bmod k$ or $b_{1}=\left(b_{2}+1\right) \bmod k$. In order to allow processors to concentrate on computational tasks and permit the overlapping of communication with computation, a router, is used for handling message communication among processors, and is usually associated with each processor. Consequently, each node consists of a Processing Element (PE) and router.

\subsection{Adaptive Wormhole Routing in the Torus Networks}

Recently, several fully adaptive routing algorithms on tori have been evaluated in [5] of which the one using Negative Hop-based (NHop) deadlock free routing augmented with a new idea called bonus cards ( $\mathrm{Nbc}$ ) has been shown to have the best performance. In [5], the Nbc routing scheme has been used in the context of Duato's methodology [6] and a routing algorithm, named Duato-Nbc with high performance and minimum virtual channel requirements was resulted. Investigations showed that Duato-Nbc has a better performance compared to other algorithms reported in the literature and the other algorithms proposed in [4]. 


\section{The Analytical Model}

In this section, we derive an analytical model for Duato-Nbc routing algorithm [5]. The most important performance measure in our model is the average message latency.

\subsection{Model Assumptions}

The model makes assumptions, which are commonly used in the literature [5, 7-11], and are listed below.

- Messages destinations are uniformly distributed across the network nodes.

- Nodes generate traffic independently of each other, following a Poisson process with an average rate of $\lambda_{g}$ messages per cycle.

- The message length is fixed at $M$ flits, each of which requires one cycle transmission time between two adjacent routers.

- The local queue at the injection channel in the source node has infinite capacity. Messages at the destination node are transferred to the local PE one at a time through the ejection channel.

- $\quad V$ virtual channels per physical channel are used. These virtual channels are used according to the Duato-Nbc routing scheme, which are divided into $V_{1}$ and $V_{2}$ classes $\left(V=V_{1}+V_{2}\right)$. This number of virtual channels yields the optimal performance compared to the other schemes proposed in [4].

In the following section, we present the mathematical model that approximates the behavior of 2-D torus communication system using Duato-Nbc routing algorithm.

\subsection{Derivation of the Model}

The average message latency is composed of the average network latency, $\bar{S}$, which is the time to cross the network and the average waiting time seen by the message in the source node, $\bar{W}_{s}$, before entering the network. However, to capture the effects of virtual channels multiplexing, the mean message latency has to be scaled by a factor, say $\bar{V}$, representing the average degree of virtual channels multiplexing, that takes place at a given physical channel. Therefore, the average message latency can be approximated as [9]

$$
\text { Average Message Latency }=\left(\bar{S}+\bar{W}_{s}\right) \bar{V}
$$

In what follows, we will describe the calculation of $\bar{S}, \bar{W}_{s}$, and $\bar{V}$.

\subsubsection{Calculation of the Mean Network Latency}

Under uniform traffic pattern, the average number of channels that a message visits along a given dimension and across the network, $\bar{k}, \bar{d}$ respectively, are given by Agarwal [10]

$$
\bar{k} \approx k / 4, \quad \bar{d}=2 \bar{k}
$$


Fully adaptive routing allows a message to use any available channel that brings it closer to its destination resulting in an evenly distributed traffic rate on all network channels. The mean arrival rate, $\lambda_{c}$, on a given channel is determined as follows. A PE generates, on average, $\lambda_{g}$ messages in a cycle, which are evenly distributed among the 4 output channels. Since each message travels, on average, $\bar{d}$ hops to cross the network, we can write the rate on a channel, $\lambda_{c}$, as

$$
\lambda_{c}=\lambda_{g} \bar{d} / 4
$$

Since the torus topology is symmetric, averaging the network latencies seen by the messages generated by only one node for all other nodes gives the average message latency in the network. Let $s=\left(s_{x}, s_{y}\right)$ be the source node and $d=\left(d_{x}, d_{y}\right)$ denote a destination node such that $d \in G-\{s\}$ where $G$ is the set of all nodes in the network. We define the set $H=\left\{h_{x}, h_{y}\right\}$, where $h_{x}$ and $h_{y}$ denote the number of hops that the message makes along $X$ and $Y$ dimensions, respectively, i.e. $\left(s_{x}+h_{x}\right) \bmod k=d_{x}$, and $\left(s_{y}+h_{y}\right) \bmod k=d_{y}$.

$$
h_{x}=\left\|s_{x}-d_{x}\right\|, \quad h_{y}=\left\|s_{y}-d_{y}\right\|
$$

where $\|x-y\|$ denotes the distance between a source node $x$ and a destination node $y$. The network latency, $S_{H}$, seen by a message crossing the network from node $s$ to node $d$ consists of two parts: the first part is the actual message transmission time, and the second part is the blocking time in the network. Therefore, $S_{H}$ can be written as

$$
S_{H}=M+\|H\|+\sum_{h=1}^{\|H\|} B_{h}
$$

where $M$ is the message length, $\|H\|$ is the distance (in terms of hops made by the message) between the source and the destination nodes, and $B_{h}$ is the blocking time seen by a message in its $h^{\text {th }}$ hop. The terms $\|H\|$ and $B_{h}$ are given by

$$
\begin{gathered}
\|H\|=h_{x}+h_{y} \\
B_{h}=P_{\text {block }_{h}} \bar{W}_{c}
\end{gathered}
$$

where $P_{\text {block }_{h}}$ is the probability that a message is blocked at its $h^{\text {th }}$ hop channel, and $\bar{W}_{c}$ is the average waiting time to acquire a virtual channel in the event of blocking. Let us now calculate the probability $P_{\text {block }_{h}}$. To do so, let $\varphi_{h}$ be the number of dimensions, or output channels, that a message still has to visit when crossing the $h^{\text {th }}$ hop channel. The calculation of $\varphi_{h}$ has been derived in [9]. We recollect briefly here the main equations for the calculation of $\varphi_{h}$. The number of channels, $\varphi_{h}$, that the message can select when crossing the $h^{\text {th }}$ hop channel, $(1 \leq h \leq \bar{d})$ is given by

$$
\varphi_{h}=\sum_{t=0}^{1}(2-t) \psi_{h}^{t}
$$

where $\psi_{h}^{t}$ is the probability that the message has entirely crossed $t(0 \leq t \leq 1)$ dimensions along on its $h$-hop path. The probability that there remains only one dimension to cross a message $h$-hops away from its destination, $P_{\varphi_{h}}$, is given by 


$$
P_{\varphi_{h}}= \begin{cases}\frac{2}{\bar{d}-h+1} & \bar{k} \leq h<\bar{d}-1 \\ 0 & 0 \leq h<\bar{k}\end{cases}
$$

Thus, the probability that the message has entirely crossed $t$ dimensions along on its $h$-hop path can be obtained by

$$
\psi_{h}^{t}= \begin{cases}1-P_{\varphi_{h}} & t=0 \\ P_{\varphi_{h}} & t=1\end{cases}
$$

A message is blocked at a given channel when all the adaptive virtual channels and also $V_{2}-\lceil\Delta / 2\rceil+1$ virtual channels of deadlock-free class (which are used for Nbc algorithm) are busy, where $\Delta$ is the number of remaining hops to reach the destination. When blocking occurs a message has to wait for all $V_{1}$ fully adaptive virtual channels, and $V_{2}-\lceil\Delta / 2\rceil+1$ deadlock-free virtual channels. In order to calculate $P_{\text {block }_{h}}$, we need to categorize messages into three classes based on their previous and next hop.

- Class 1: This class contains messages which have used a virtual channel of fully adaptive class in their previous hop (with probability $\mathrm{P}_{\text {block }}$ ).

- Class 2: This class contains messages which have used a virtual channel of deadlock-free class in their previous hop and their next hop is negative (with probability $\mathrm{P}_{\text {block }_{2}}$ ).

- Class 3: This class contains messages which have used a virtual channel of deadlock-free class in their previous hop and their next hop is positive (with probability $\mathrm{P}_{\text {block }_{3}}$ ).

Since the number of messages in Class 2 is identical to the number of messages in Class 3, we can write the probability of message blocking as

$$
P_{\text {block }}=\left(P_{\text {block } 1}+\left(P_{\text {block } 2}+P_{\text {block } 3}\right) / 2\right)^{\varphi_{h}}
$$

In what follows, we compute $\mathrm{P}_{\text {block }_{1}}, \mathrm{P}_{\text {block }_{2}}$, and $\mathrm{P}_{\text {block }}$.

When a message used a virtual channel of fully adaptive class in its previous hop, it can use any of $V_{1}$ virtual channels of this class, and also $V_{2}-\lceil\Delta / 2\rceil+1$ virtual channels of deadlock-free class. The blocking occurs, when all $V_{1}+V_{2}-\lceil\Delta / 2\rceil+1$ virtual channels at a given physical channel are busy. We can therefore write

$$
\mathrm{P}_{\text {block }_{1}}=\sum_{v=V_{1}+V_{2}-\lceil\Delta / 2\rceil+1}^{V} P_{v_{0}} P_{v}\left(\begin{array}{c}
\lceil\Delta / 2\rceil-1 \\
v-V_{1}-V_{2}+\lceil\Delta / 2\rceil-1
\end{array}\right) /\left(\begin{array}{c}
v \\
v
\end{array}\right)
$$

where $P_{v},(0 \leq v \leq V)$, and $P_{v_{0}}$ denote the probability that $v$ virtual channels at a physical channel are busy (which is calculated later in Section 3.2.3), and the probability 
that the message uses a virtual channel of Class $a$ in its previous hop, respectively. $P_{v_{0}}$ is given by

$$
P_{v_{0}}=V_{1} /\left(V_{1}+V_{2}-\lceil\Delta / 2\rceil+1\right)
$$

Moreover, a message belonging to the Classes 2 and 3 which has already used virtual channel $l$ at its previous hop, can employ any of $V_{1}$ virtual channels of fully adaptive class, and also $V_{2}-\lceil\Delta / 2\rceil-l+1$ and $V_{2}-\lceil\Delta / 2\rceil-l+2$ virtual channels of deadlockfree class, respectively. Therefore, the $\mathrm{P}_{\text {block } 2}$ and $\mathrm{P}_{\text {block } 3}$ are obtained by

$$
\begin{aligned}
& \mathrm{P}_{\text {block } 2}=\sum_{l=1}^{\left.V_{2}-\Delta \Delta / 2\right\rceil} \sum_{v=V_{1}\lceil\lceil\Delta / 2\rceil+l}^{V} P_{v_{l}} P_{v}\left(\begin{array}{c}
v_{2}\lceil\lceil\Delta / 2\rceil-l \\
v-V_{1}\lceil-\lceil/ 2\rceil-l
\end{array}\right) /\left(\begin{array}{c}
v \\
v
\end{array}\right) \\
& \mathrm{P}_{\text {block } 3}=\sum_{l=1}^{V_{2}\lceil-\lceil/ 2\rceil+1} \sum_{v=V_{1}\lceil\lceil\Delta / 2\rceil+l+1}^{v} P_{v l} P_{v}\left(\begin{array}{c}
V_{2}-\lceil\Delta / 2\rceil-l-1 \\
v-V_{1}\lceil\Delta / 2\rceil-l-1
\end{array}\right) /\left(\begin{array}{c}
v \\
v
\end{array}\right)
\end{aligned}
$$

where $P_{v_{l}}$ indicates the probability that a message has used virtual channel $l$ at its previous hop and is given by

$$
P_{v_{l}}=1 /\left(V_{1}+V_{2}-\lceil\Delta / 2\rceil+1\right) \quad 1 \leq l \leq V_{2}-\lceil\Delta / 2\rceil+1
$$

The average network latency, $\bar{S}$, is obtained by averaging $S_{H}$, the average network latency of $H$-hops messages, over the $(N-1)$ possible destination nodes in the network. Therefore, $\bar{S}$ can be determined as

$$
\bar{S}=\frac{1}{N-1} \sum_{d \in G-\{s\}} S_{H}
$$

To determine the average waiting time to acquire a virtual channel, a physical channel may be treated as an M/G/1 queue [8]. Since the minimum service time at a channel is equal to the message length, $M$, following a suggestion proposed in [11], the variance of the service time distribution can be approximated by $(\bar{S}-M)^{2}$; where $\bar{S}$ is the average service time at a given channel and can be calculated as the mean of $S_{H}$ of all source and destination nodes that have at least one path between each other that traverse the channel. Hence, the average waiting time becomes

$$
\bar{W}_{c}=\lambda_{c} \bar{S}^{2}\left(1+(\bar{S}-M)^{2} / \bar{S}^{2}\right) /\left(2\left(1-\lambda_{c} \bar{S}\right)\right)
$$

\subsubsection{Calculation of the Average Waiting Time in the Source Node}

A message originating from a given source node sees a network latency of $\bar{S}$. Since a message in the source node can enter the network through any of the $V$ virtual channels, the average arrival rate to the queue is $\lambda_{g} / V$. Applying the Pollaczek-Khinchine $(\mathrm{P}-\mathrm{K})$ mean value formula [8] yields the average waiting time experienced by a message at the source node as [8] 


$$
\bar{W}_{s}=\left(\lambda_{g} / V\right) \bar{S}^{2}\left(1+(\bar{S}-M)^{2} / \bar{S}^{2}\right) /\left(2\left(1-\left(\lambda_{g} / V\right) \bar{S}\right)\right)
$$

\subsubsection{Calculation of Average Multiplexing Degree of Virtual Channels}

The probability, $P_{v}(0 \leq v \leq V)$, that $v$ virtual channels at a given physical channel are busy can be determined using a Markovian model (details of the model can be found in $[7,9])$. In the steady state, the model yields the following probabilities [7].

$$
Q_{v}=\left\{\begin{array}{lr}
1 & v=0 \\
Q_{v-1} \lambda_{c} \bar{S} & 0<v<V \\
Q_{v-1} \lambda_{c} /\left(1 / \bar{S}-\lambda_{c}\right) & v=V
\end{array} \quad P_{v}=\left\{\begin{array}{lr}
\left(\sum_{v=0}^{V} Q_{v}\right)^{-1} & v=0 \\
P_{v-1} \lambda_{c} \bar{S} & 0<v<V \\
P_{v-1} \lambda_{c} /\left(1 / \bar{S}-\lambda_{c}\right) & v=V
\end{array}\right.\right.
$$

When multiple virtual channels are used per physical channel they share the bandwidth in a time multiplexed manner. The average degree of virtual channel multiplexing, that takes place at a given physical channel, can be estimated by [7]

$$
\bar{V}=\sum_{v=1}^{V} v^{2} P_{v} / \sum_{v=1}^{V} v P_{v}
$$
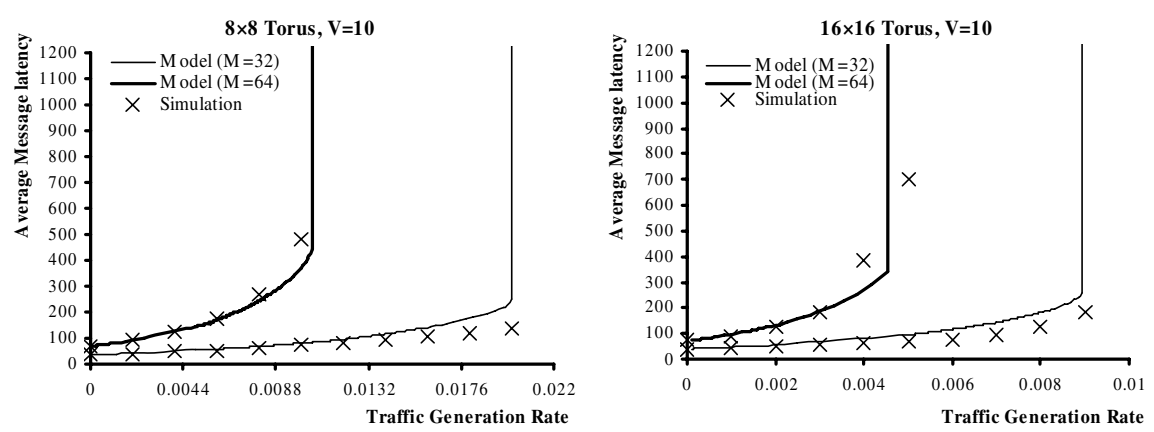

Fig. 1. Average message latency calculated by the analytical model against those obtained through simulation for $8 \times 8$ and $16 \times 16$ torus networks using Duato-Nbc routing with message length $M=32,64$ flits, and $V=10$ virtual channels per physical channel

\section{Simulation Results}

To further understand and evaluate the performance issues of the routing algorithm, we have developed an event-driven simulator at flit-level. The average message latency is defined as the average amount of time from the generation of a message until the last data flit reaches the local PE at the destination. We ran each simulation for 300,000 flit times and sufficient warm-up times (through discarding the information obtained during the first 10,000 flit times) is provided to allow the network to reach the steady state. Numerous experiments are conducted for different sizes of the network, virtual channels, and message lengths to assess the accuracy of the analytical model. Fig. 1 depicts latency results predicted by the analytical model plotted against 
those provided by the simulator for 2-D $8 \times 8$ and $16 \times 16$ torus networks, respectively; with $V=10$ virtual channels per physical channel, and different message lengths, $M=32,64$ flits. The $x$-axis in this figure represents the traffic rate injected by a given node in a cycle $\left(\lambda_{g}\right)$ while the $y$-axis shows the average message latency (in flit cycles). The figure indicates that the analytical model predicts the mean message latency with a good degree of accuracy in all regions. However, some discrepancies around the saturation point are apparent. This is a result of the approximations made when constructing the analytical model, e.g. the approximation used to estimate the variance of the service time distribution at a channel. This approximation greatly simplifies the model by avoiding the computation of the exact distribution of the message service time at a given channel.

\section{Conclusions}

In this paper, we proposed a mathematical performance model to predict the average message latency in wormhole-switched 2-D tori using the fully adaptive scheme proposed in [5]. Simulation experiments have revealed that the message latency results predicted by the analytical model are in good agreement with those obtained through simulation under different working conditions. We showed that the proposed model manages to achieve a good degree of accuracy while maintaining simplicity, making it a practical evaluation tool that can be used by the researchers in the field to gain insight into the performance behavior of fully adaptive routings in wormhole-switched torus networks. Our next objective is to develop an analytical modeling approach to investigate the performance behavior of this routing scheme in the presence of failures.

\section{References}

1. Duato, J., Yalamanchili, S., Ni, L.M: Interconnection networks: An engineering approach, Morgan Kaufmann Publishers, New York (2003).

2. Ni, L. M., McKinley, P. K.: A Survey Of Wormhole Routing Techniques in Direct Networks, IEEE Computer Society Press, 26 (2) (1993) 62-76.

3. Dally, W. J., Aoki, H.: Deadlock-free adaptive routing in multicomputer networks using virtual channels, IEEE TPDS, 4 (4) (1993) 66-74.

4. Boppana, R.V., Chalasani, S.: A Framework for Designing Deadlock-Free Wormhole Routing Algorithms, IEEE TPDS, 7 (2) (1996) 169-183.

5. Safaei, F., et al.: Performance Comparison Of Routing Algorithms In Wormhole-Switched Fault-Tolerant Interconnect Networks, International Conference on Network and Parallel Computing (NPC06), Japan, October 2006.

6. Duato, J.: A New Theory of Deadlock-Free Adaptive Routing in Wormhole Routing Networks, IEEE TPDS, 4 (12) (1993) 320-1331.

7. Dally, W. J.: Virtual Channel Flow Control, IEEE TPDS, 3 (2) (1992) 194-205.

8. Kleinrock, L.: Queuing Systems, Vol. 1, John Wiley, New York (1975).

9. Ould-Khaoua, M.: A Performance Model of Duato's Adaptive Routing Algorithm in k-ary n-cubes, IEEE TC, 48 (12) (1999) 1-8.

10. Agarwal, A.: Limits on Interconnection Network Performance, IEEE TPDS, 2 (4) (1991) 398-412.

11. Draper, J.T., Ghosh, J.: A Comprehensive Analytical Model for Wormhole Routing in Multicomputer Systems, JPDC, 32 (2) (1994) 202-214. 